\title{
OPEN Predicting clinical outcomes of cancer patients with a p53 deficiency gene signature
}

\author{
Evelien Schaafsma ${ }^{1}$, Eric M. Takacs ${ }^{2}$, Sandeep Kaur ${ }^{2}$, Chao Cheng ${ }^{1,3 凶}$ \& \\ Manabu Kurokawa $\mathbb{B}^{2}$
}

The tumor suppressor p53, encoded by the TP53 gene, is mutated or nullified in nearly $50 \%$ of human cancers. It has long been debated whether TP53 mutations can be utilized as a biomarker to predict clinical outcomes of cancer patients. In this study, we applied computational methods to calculate p53 deficiency scores (PDSs) that reflect the inactivation of the p53 pathway, instead of TP53 mutation status. Compared to TP53 mutation status, the p53 deficiency gene signature is a powerful predictor of overall survival and drug sensitivity in a variety of cancer types and treatments. Interestingly, the PDSs predicted clinical outcomes more accurately than drug sensitivity in cell lines, suggesting that tumor heterogeneity and/or tumor microenvironment may play an important role in predicting clinical outcomes using p53 deficiency gene signatures.

The tumor suppressor p53, also known as the guardian of the genome, is a transcription factor encoded by the TP53 gene in humans. It acts as a sensor of cellular homeostasis and plays important roles in DNA repair, senescence, metabolism, and induction of apoptotic and non-apoptotic cell death in order to maintain the integrity of the genome and normal cellular functions. It can activate a myriad of target genes involved in cell cycle arrest, metabolism, and cell death in response to DNA damage, hypoxia, oxidative stress, and DNA mutations, resulting in the restriction of tumor development ${ }^{1}$. Considering its importance in key cellular processes, TP53 is found to be mutated in $\sim 50 \%$ of human cancers, more than any other gene in human cancers ${ }^{2}$. In this regard, Li-Fraumeni syndrome is a familial disorder that is associated with a TP53 mutation which predisposes carriers to a broad spectrum of cancers ${ }^{3}$. Also, a number of knockout mouse studies showed that the loss of the TP53 gene leads to the development of spontaneous tumors, particularly sarcoma and lymphoma ${ }^{4}$.

In the past decades, much effort has been focused on predicting clinical outcomes based on TP53 status in a variety of cancers. Some studies have successfully shown that TP53 mutations/deletion are linked to a poorer prognosis in certain cancer types, such as head and neck, liver, and hematopoietic cancers ${ }^{5}$. However, numerous conflicting results have been reported for cancers of the bladder, brain, breast, lung, colon, esophagus, and ovary ${ }^{5}$. For instance, there were approximately 1157 reports on lung and bronchus cancers showing that TP53 mutations were associated with poor survival, while 1167 reports showed no such association ${ }^{5}$. Therefore, based on these studies, it appears that no simple generalization can be made regarding the association of TP53 mutation status with clinical outcomes. Importantly, it is known that some of the p53 mutants, if not all, show gain-of-function rather than loss-of-function mutations, indicating that p53 mutants can induce genes that wild type p53 cannot control. Supporting this, recent studies suggest that the prognostic value of the TP53 mutation may depend on the mutated residue $e^{6,7}$, cancer type, presence of mutation in other genes ${ }^{8}$, and clinical backgrounds ${ }^{9}$. Most importantly, TP53 mutation status is not the only factor affecting the p53 signaling pathway. Even if cancer cells carry wild type p53, its activity is often suppressed upstream by negative regulators, such as MDM2 and MDM $4^{10,11}$, or downstream by promoter methylation of p53 target genes ${ }^{12,13}$.

Given the complexity of the regulation of p53 and its target expression, p53 activity is expected to be a better prognostic marker than TP53 mutation status. Our group has previously demonstrated that the p53 deficiency score (PDS), which is inferred based on expression levels of p53 regulated genes, is a better predictor of recurrence in early-stage lung adenocarcinoma patients than TP53 mutation status ${ }^{14}$. In the present study, we extended the computational approach to include 20 cancer types to analyze the association between PDS and clinical outcomes.

\footnotetext{
${ }^{1}$ Department of Biomedical Data Science, Geisel School of Medicine at Dartmouth, Hanover, NH 03755, USA. ${ }^{2}$ Department of Biological Sciences, Kent State University, Kent, OH 44242, USA. ${ }^{3}$ Department of Medicine, Baylor College of Medicine, Houston, TX 77030, USA. ${ }^{\circledR}$ email: Chao.Cheng@bcm.edu; mkurokaw@kent.edu
} 


\begin{tabular}{|l|l|l|l|l|}
\hline Cancers & \# Samples & \# TP53 mutant & \# TP53 wild type & \% Mutation \\
\hline OV & 206 & 192 & 14 & 93.2 \\
\hline UCS & 57 & 52 & 5 & 91.2 \\
\hline ESCA & 184 & 158 & 26 & 85.9 \\
\hline LUSC & 482 & 403 & 79 & 83.6 \\
\hline READ & 146 & 107 & 39 & 73.3 \\
\hline HNSC & 501 & 353 & 148 & 70.5 \\
\hline PAAD & 169 & 104 & 65 & 61.5 \\
\hline COAD & 405 & 223 & 182 & 55.1 \\
\hline LUAD & 512 & 260 & 252 & 50.8 \\
\hline LGG & 524 & 257 & 267 & 49.0 \\
\hline STAD & 411 & 201 & 210 & 48.9 \\
\hline BLCA & 406 & 198 & 208 & 48.8 \\
\hline UCEC & 529 & 203 & 326 & 38.4 \\
\hline SARC & 237 & 86 & 151 & 36.3 \\
\hline GBM & 158 & 55 & 103 & 34.8 \\
\hline BRCA & 1023 & 349 & 674 & 34.1 \\
\hline KICH & 66 & 21 & 45 & 31.8 \\
\hline LIHC & 360 & 108 & 252 & 30.0 \\
\hline SKCM & 467 & 72 & 595 & 11.9 \\
\hline PRAD & 494 & 59 & & \\
\hline
\end{tabular}

Table 1. TP53 status of each cancer types.

\section{Results}

We have previously proposed a computational method to predict sample-specific PDS in lung adenocarcinoma based on the expression of genes that are associated with TP53 gene mutation status ${ }^{14}$. Here, we extended this method to 19 additional cancer types from The Cancer Genome Atlas (TCGA). These cancer types were selected based on having at least 20 patients who had protein-altering TP53 mutations and made up at least 10\% of all patients within a cancer type. The total number of patients with TP53 mutations in these 20 cancer types was 3461 , which constituted $47 \%$ of all included samples (Table 1). Cancer-specific mutation rates ranged from $93 \%$ for ovarian cancer to $12 \%$ for prostate cancer (Table 1). For each cancer type, we generated p53 deficiency signatures in which genes are weighted based on their association with TP53 mutation status; genes highly associated with TP53 mutations receive high weights, whereas uncorrelated genes receive small weights. The resulting weight profiles were mildly but positively correlated (Fig. 1A), with the highest correlation being between the rectum adenocarcinoma and colon adenocarcinoma signatures (Spearman Correlation Coefficient $=0.583, \mathrm{p}<2 \mathrm{E}-323$ ).

The percentage of surviving patients is high for most TCGA cancer types, which makes it difficult to identify prognostic associations due to low statistical power. We selected cancer types with a relatively high death event percentage $(>50 \%)$. Within these cancer types, we observed significant differences in survival, including uterine corpus endometrial carcinoma $(\log$-rank $\mathrm{p}=0.003)$ and lung adenocarcinoma $(\log$-rank $\mathrm{p}=0.008)($ Fig. $1 \mathrm{~B}, \mathrm{C})$. Interestingly, there was no statistical association between overall survival and TP53 mutation status in these cancer types (Fig. 1B,C). In fact, TP53 mutation status was only prognostic in pancreatic adenocarcinoma (log-rank $\mathrm{p}=2 \mathrm{E}-3$ ). To more comprehensively evaluate the association between PDS and patient prognosis, we also utilized the PRECOG dataset ${ }^{15}$, a comprehensive collection of more than 100 gene expression datasets with high-quality survival data from patients treated with standard-of-care chemotherapy. We confirmed the prognostic relevance of PDS in uterine corpus endometrial carcinoma and lung adenocarcinoma and found a number of additional cancer types in which p53 deficiency was associated with prognosis. Generally, high PDSs were associated with shorter survival; high PDSs were hazardous in patients with prostate adenocarcinoma, bladder urothelial carcinoma, breast invasive carcinoma, liver hepatocellular carcinoma, and head and neck squamous carcinoma (Fig. 1D). Interestingly, brain cancers including glioblastoma multiforme and brain lower grade glioma showed a negative association with survival, as well as ovarian serous cystadenocarcinoma (Fig. 1D). These findings suggest that under standard-of-care chemotherapy, p53 deficiency is associated with poorer survival outcomes in about half of the evaluated cancer types; exceptions were observed in brain cancers in which p53 deficiency is associated with improved survival.

Next, we examined whether PDSs can predict drug sensitivity in cancer cell lines. Towards this end, we inferred PDSs in the Cancer Cell Line Encyclopedia (CCLE) dataset which contains drug sensitivity data on different drugs in a wide range of cell lines ${ }^{16}$. We could only confidently capture p53 deficiency in a limited number of cancer types (AUC of predicting TP53 mutation status $>0.60$ ) (Fig. 2A). We thus evaluated associations between PDSs and drug sensitivity in five cancer types for which we could confidently capture p53 deficiency (breast invasive carcinoma, colon adenocarcinoma, ovarian serous cystadenocarcinoma, pancreatic adenocarcinoma, and skin cutaneous melanoma). We also only included drugs for which at least 10 cell lines for the given cancer type were tested, resulting in the evaluation of 11 drugs for breast invasive carcinoma and up to 15 drugs 

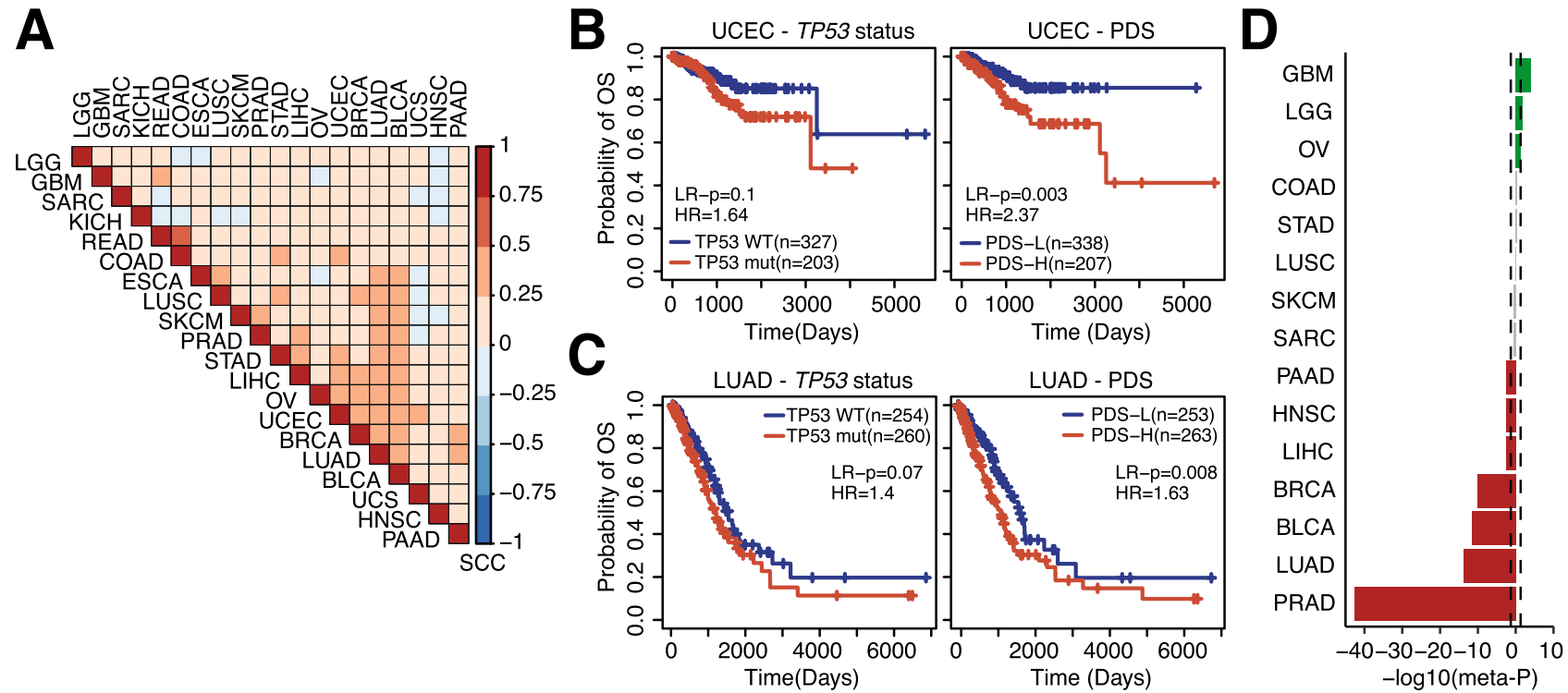

Figure 1. Prognostic associations in TCGA and PRECOG. (A) Correlation matrix of TP53 signatures using Spearman correlation coefficients (SCC). (B) KM plots for UCEC in TCGA based on TP53 mutation status (left) or p53 deficiency scores (PDS) (right). (C) KM plots for LUAD in TCGA based on TP53 mutation status (left) or PDS (right). LR p-value calculated between groups using Log-rang tests, HR hazard ratio. (D) Metap-values for association between PDSs and prognosis in PRECOG. Bars indicate-log10(meta p-values). Negative values indicate a hazardous role for PDSs, while positive values represent protective associations. LGG (brain lower grade glioma), GBM (glioblastoma multiforme), SARC (sarcoma), KICH (kidney chromophobe), READ (rectum adenocarcinoma), COAD (colon adenocarcinoma), ESCA (esophageal carcinoma), LUSC (lung squamous cell carcinoma), SKCM (skin cutaneous melanoma), PRAD (prostate adenocarcinoma), STAD (stomach adenocarcinoma), LIHC (liver hepatocellular carcinoma), OV (ovarian serous cystadenocarcinoma), UCEC (uterine corpus endometrial carcinoma), BRCA (breast invasive carcinoma), LUAD (lung adenocarcinoma), BLCA (bladder urothelial carcinoma), UCS (uterine carcinosarcoma), HNSC (head and neck squamous cell carcinoma), PAAD (pancreatic adenocarcinoma).

A

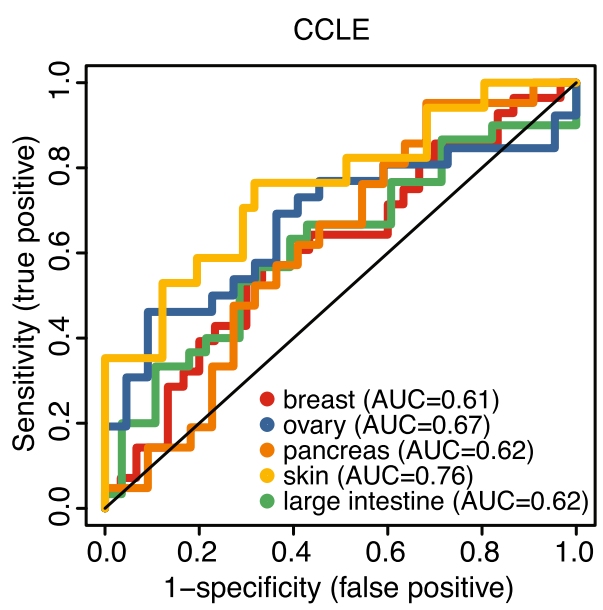

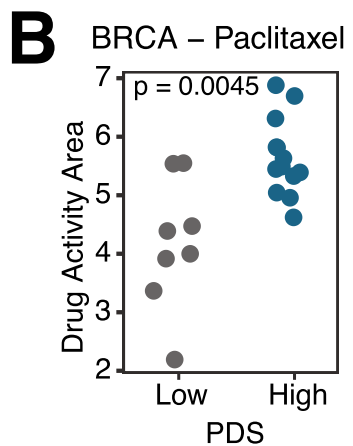

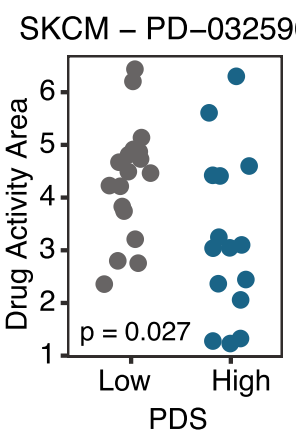

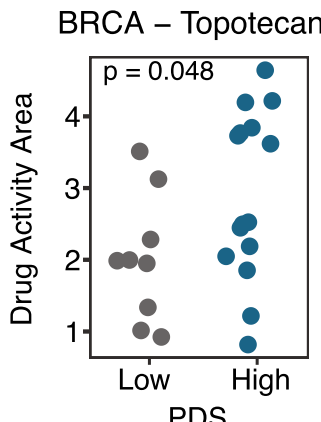

COAD - PD-0325901
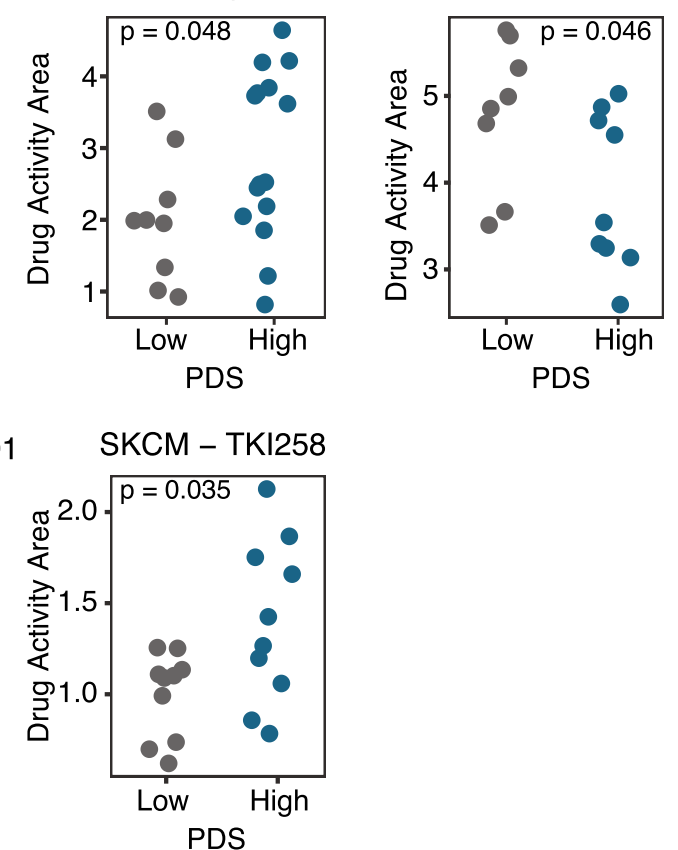

Figure 2. Drug sensitivity in CCLE data. (A) AUC curves of PDS predicting TP53 mutation status for cancer types with AUC scores $>0.60$. (B) Differences in drug sensitivity based on PDS status using the median of PDSs to demarcate low and high p53 deficiency groups. p-value calculated by two-sided Student's t-test. 
for skin cutaneous melanoma. A small number of evaluated associations was statistically significant (Fig. 2B). In two out of five cases (colon adenocarcinoma and skin cutaneous melanoma), p53 deficiency was associated with increased drug sensitivity, whereas in the other three comparisons (breast invasive carcinoma, ovarian serous cystadenocarcinoma, and pancreatic adenocarcinoma), p53 deficiency was associated with decreased drug sensitivity (Fig. 2B).

To further investigate the relationship between the TP53 gene expression signature and drug sensitivity at the cell level, we analyzed p53 deficiency and paclitaxel sensitivity by an actual cell culture model of breast cancer where a strong inverse association between p53 deficiency and paclitaxel sensitivity was observed (Fig. 2B). We treated 9 breast cancer cell lines endogenously expressing a mutant p53 (MDA-MB-468, HCC-1954, MDAMB-231, AU-565, SKBR-3, HCC-202, BT-474, HCC1419, and T47D) with various concentrations of paclitaxel in vitro. Forty-eight hours later, we performed MTS assays and calculated paclitaxel IC50's for each cell line (Fig. 3A). Supporting the CCLE dataset analysis (Fig. 2B), the MDA-MB-468 and MDA-MB-231 cell lines, which showed high PDSs, were highly resistant to paclitaxel (Fig. 3A,B). In contrast, the HCC-1954 cell line was sensitive to paclitaxel (Fig. 3A), even though it had a high PDS (Fig. 3B). Conversely, the HCC-1419 cell line, which showed a low PDS (Fig. 3B), was resistant to paclitaxel (Fig. 3A). Overall, among the 9 cell lines tested, there was a mild association between high PDSs and paclitaxel resistance in vitro (Fig. 3B), with 2 out of 4 cell lines in the high PDS category being resistant to paclitaxel and only 1 out of 5 cell lines being resistant in the low PDS category. These results suggest that PDSs can be used as a prognostic marker for clinical samples and in some cell lines. The lower association between PDSs and treatment response in cell lines could be due to the inherent differences between gene expression profiles of tumor tissues and cell lines, including the absence of surrounding stromal cells and immune cells as well as the lack of substantial tumor cell heterogeneity in cell lines. In addition, some cell lines are equipped with mechanistic aspects that could cause drug resistance independently of p53 activity. For example, the breast cancer cell line MCF7 expresses wild type p53. However, the lack of caspase- 3 makes this cell line extremely resistant to cell death stimuli, including paclitaxel, despite its low PDS (score-203).

Cancer patients often undergo different treatment schemes. To evaluate the effect of different treatment modalities in tumor gene expression data, we applied the breast cancer-specific PDS to the METABRIC (Curtis) datase $^{17}$, which contains gene expression data from patients treated by a variety of therapies. Overall, PDSs were hazardous (Fig. 4A), consistent with our earlier findings in Fig. 1. When comparing low and high PDSs in patients treated with the same therapy, significant differences in survival were observed; p53 deficiency was always associated with poor prognosis. This association reached statistical significance for the chemotherapy/ hormone therapy/radiotherapy combination (log-rank $\mathrm{p}=0.002)($ Fig. $4 \mathrm{~B})$, the hormone therapy/radiotherapy combination (log-rank $\mathrm{p}=3 \mathrm{E}-4)($ Fig. $4 \mathrm{C})$ and for patients that did not receive any therapy $(\log$-rank $\mathrm{p}=0.02)$ (Fig. 4D). No significant relationship was observed within the patient group treated with only chemotherapy (data not shown). Consistent with our earlier findings (Fig. 1B,C), p53 deficiency was a better predictor than TP53 mutation status (Fig. 4A-D).

The comparison of the survival probability among treatments also revealed differential survival among patient groups treated with different therapies. For example, patients receiving a combination of chemotherapy/ hormone therapy/radiotherapy survived much longer compared to patients who only received chemotherapy (Fig. 5A). Interestingly, some treatment groups were associated with PDSs. For instance, the chemotherapy (CT) and chemotherapy/radiotherapy (CT/RT) groups of patients had higher PDSs as compared to other treatment groups (Fig. 5B). Since patients within this dataset were not randomized into treatment groups, it is possible that breast cancer subtypes contribute to this discrepancy as subtypes play a major role in determining treatment options. We found that basal and HER2+ breast cancer patients have much higher PDSs compared to the other subtypes (Fig. 5C) and these are the breast cancer subtypes with the poorest survival ${ }^{18}$. Compared to basal and HER2 + breast cancers, the Luminal A, Luminal B, and normal-like subtypes are known to be associated with better prognosis. Notably, PDSs predicted patients' survival within these subtypes (Fig. 5D).

\section{Discussion}

Gene expression signatures are emerging as powerful prognostic markers of clinical outcomes as compared to mutation status of a small gene set. Previously, we have generated a computational p53 deficiency gene signature model $^{14}$. In the present study, we sought to investigate whether the PDS also predicts clinical outcomes in other cancer types. We have shown that PDS is a better predictor of clinical outcomes as compared to TP53 mutation status. It is important to note that the TP53 gene expression signature can be influenced by transcription factors other than p53. For instance, although p53 is considered to be the primary transcription factor for this signature, the p53 family proteins p63 and p73 are also known to induce the expression of many p53-targeted genes ${ }^{19-21}$. This may at least partially explain why cell lines expressing mutant p53 had a range of PDSs (Fig. 3). In addition, clinical tumor samples likely contain non-cancer cells, such as stromal cells and immune cells, which would also affect PDSs as discussed below.

Despite high survival rates in TCGA datasets, which limits the overall statistical power of prognostic analysis, we found a significant difference in overall survival in uterine corpus endometrial carcinoma and lung adenocarcinoma, where high PDSs predicted poor overall survival (Fig. 1B). Likewise, in the PRECOG dataset, high PDSs were found to be associated with poor survival in patients with prostate adenocarcinoma, lung adenocarcinoma, bladder urothelial carcinoma, breast invasive carcinoma, liver hepatocellular carcinoma, head and neck squamous carcinoma, and pancreatic adenocarcinoma (Fig. 1C). These results are in accordance with other studies in which TP53 gene mutation/signatures were shown to be predictive of clinical outcomes in different types of cancer ${ }^{18,22-26}$. Surprisingly, in glioblastoma multiforme, brain lower grade glioma, and ovarian serous cystadenocarcinoma, high PDSs predicted better survival under chemotherapy (Fig. 1C). Recently, it was reported that TP53 mutation status alone is associated with improved survival in glioblastoma ${ }^{27}$. Our results support 
A

MDA-MB-468

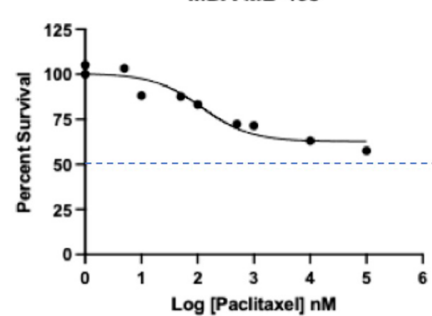

AU-565

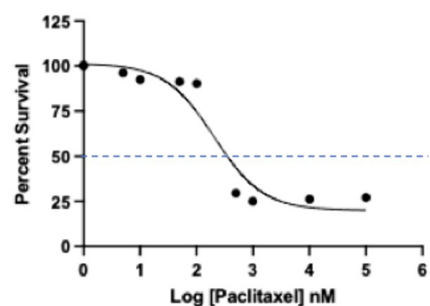

BT-474

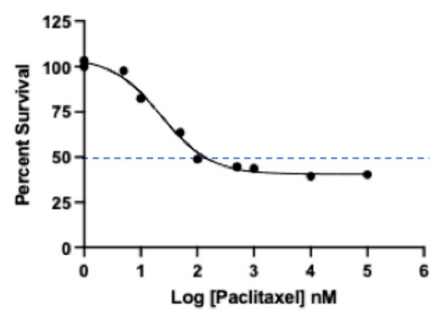

HCC-1954

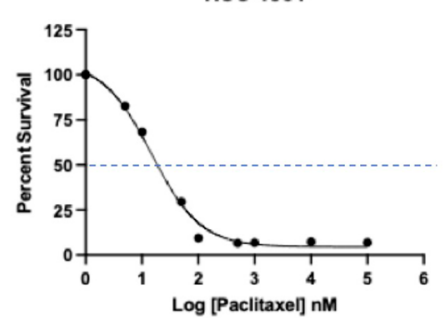

SKBR-3

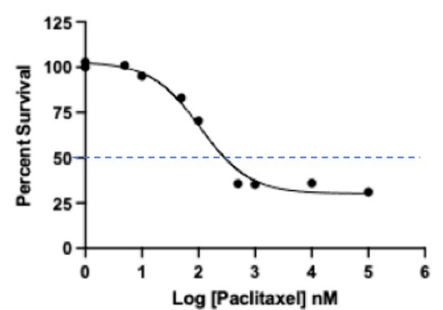

HCC-1419

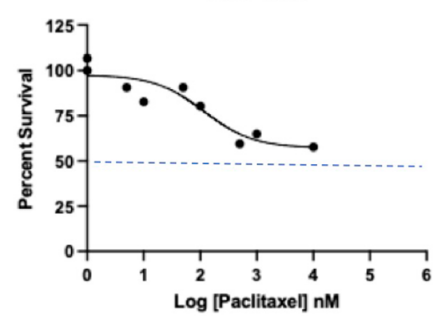

MDA-MB-231

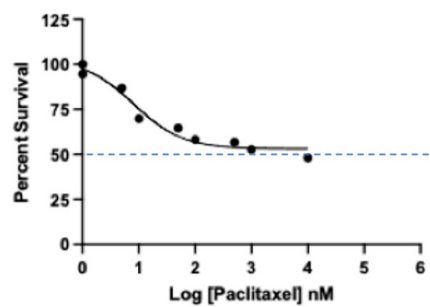

HCC-202

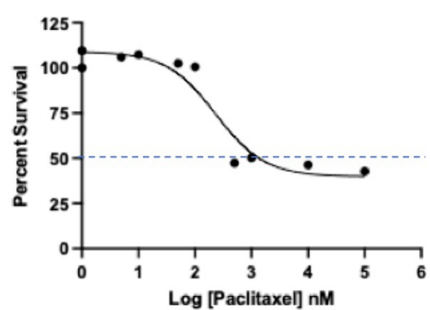

T47D

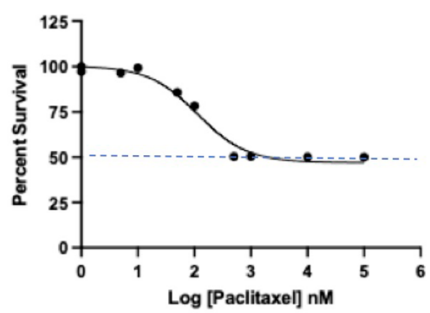

B

\begin{tabular}{|c|c|c|c|}
\hline & \multicolumn{2}{|c|}{ PDS } & IC50 (uM) \\
\hline MDA-MB-468 & 18.97 & \multirow{4}{*}{ High } & $>10$ \\
\hline HCC-1954 & 13.3 & & 0.02 \\
\hline MDA-MB-231 & 7.66 & & $>10$ \\
\hline$A U-565$ & 4.31 & & 0.35 \\
\hline SKBR-3 & 0.14 & \multirow{5}{*}{ Low } & 0.29 \\
\hline HCC-202 & -67 & & 1.35 \\
\hline BТ-474 & -114 & & 0.13 \\
\hline HCC-1419 & -210 & & $>10$ \\
\hline T47D & -219 & & 2.7 \\
\hline
\end{tabular}

Figure 3. Drug sensitivity in breast cancer cell lines. (A) The indicated breast cancer cell lines were plated into 96-well plates and were treated with various concentrations of paclitaxel. MTS assays were performed following a 48-h incubation with paclitaxel. (B) PDSs and paclitaxel IC50's are shown for each breast cancer cell line.

this observation. It is interesting to note that the frequency of TP53 mutations vary among these cancer types (Table 1). The functional significance of p53 and its mutation in glioblastoma, low-grade glioma, and ovarian cancer needs to be further investigated. 
A

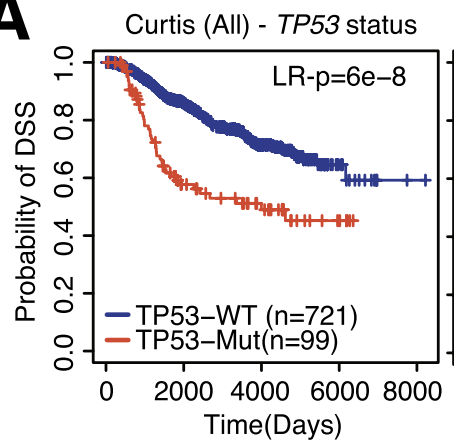

C

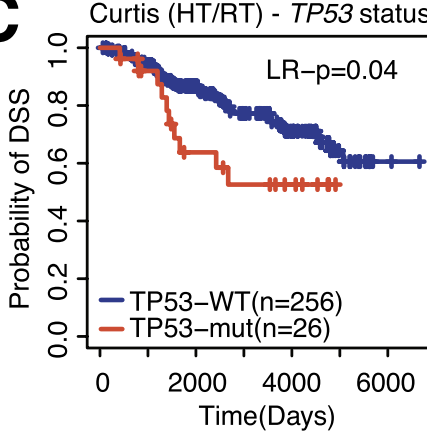

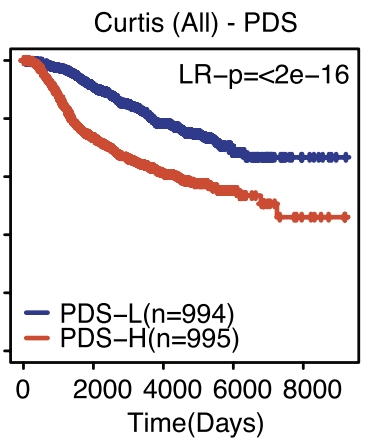

Curtis (HT/RT) - PDS

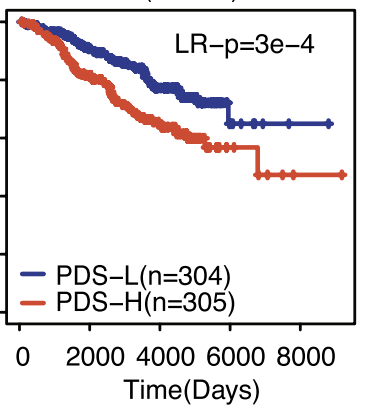

B
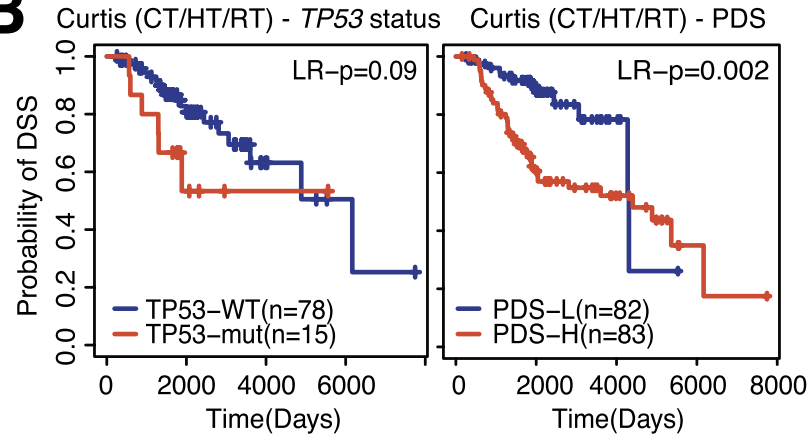

D

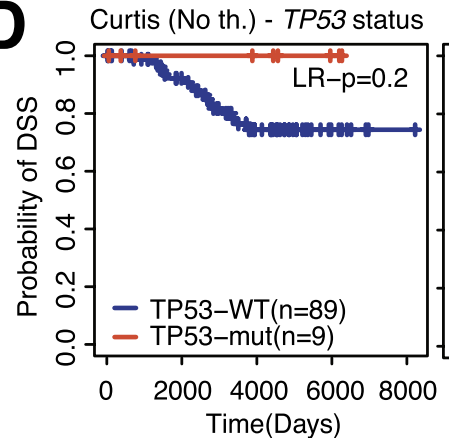

Curtis (No th.) - PDS

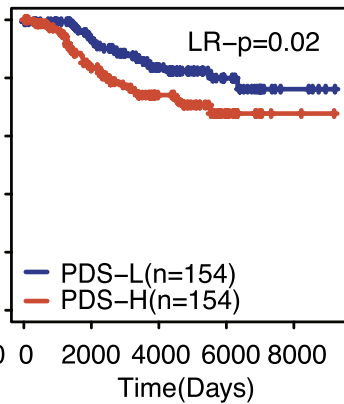

Figure 4. PDSs in the METABRIC/Curtis dataset. (A) KM plot evaluating the association of TP53 mutation status (left) or PDS (right) with survival in the METABRIC/Curtis dataset (all patients). (B) Association of TP53 mutation status (left) or PDS (right) with survival for patients receiving a combination of chemotherapy therapy, hormone therapy, and radiotherapy. (C) Association of TP53 mutation status (left) or PDS (right) with survival for patients receiving a combination of hormone therapy and radiotherapy. (D) Association of TP53 mutation status (left) or PDS (right) with survival for patients not receiving any treatment. $L R$ p-value calculated between TP53 groups using Log-rang tests, Cox- $p$ p-value calculated using Coxph regression using PDSs as continuous variable, DSS disease-specific survival.

Our PDS model was also validated in breast cancer subtypes. The TP53 gene mutation frequency ranges from $12 \%$ in Luminal A, 32\% in luminal B, 75\% in HER2-enriched breast cancers to $84 \%$ in basal-like cancers ${ }^{18}$. Indeed, our analysis indicated that the PDSs were high in basal-like and HER2+ breast cancer patients, while patients with the Luminal A subtype showed the lowest scores (Fig. 4C). It is worth noting that basal-like and HER2-enriched breast cancer subtypes are known to be associated with the worst survival rates ${ }^{18}$. Unfortunately, patients within the METABRIC/Curtis dataset were not treated with Herceptin therapy, the most common therapy for HER2+ breast cancer patients, preventing conclusions about the association between PDSs and response to Herceptin. Nevertheless, we were able to scrutinize the impact of PDSs in other therapies based on the available data. It was found that high deficiency scores predicted poor survival in patients treated with the chemotherapy/hormone therapy/radiotherapy combination (CT/HT/RT) (Fig. 4B), the hormone therapy/ radiotherapy combination (HT/RT) (Fig. 4C), and for patients who did not receive any therapy (Fig. 4D).

While PDSs were associated with treatment response in breast cancer, we noted that the relationship between p53 deficiency and treatment sensitivity was complex. A recent comprehensive analysis revealed that although mutant p53 can impact clinical outcomes negatively, wild type p53 could also mediate poor clinical responses depending on the treatments and breast cancer subtype ${ }^{28}$. It has been reported that a 39 -gene p53 signature is predictive of poor prognosis after adjuvant tamoxifen therapy in ER+, but not ER-, breast cancer ${ }^{29}$. In a neoadjuvant chemotherapy setting however, TP53 mutation status is associated with better patient outcomes in earlystage breast cancer ${ }^{30-33}$. In basal-like breast cancer, p53 deficiency predicts a better response to an epirubicincyclophosphamide regimen $^{34}$. Thus, additional variables related to p53 deficiency likely determine treatment sensitivity. One suggested cause is that certain therapies, including DNA-damaging reagents, induce senescence in p53-proficient tumors, which may, in turn, lead to the emergence of the persister cells that secrete senescenceassociated cytokines and, thereby, promote tumor cell survival ${ }^{35,36}$. In this regard, it is interesting to note that PDSs predicted clinical outcomes more accurately than drug sensitivity at the cell line level (Fig. 3). This could be partially ascribed to cell lines' intrinsic resistance to cell death that operates independently of the p53 pathway. Another possibility may be explained by the fact that cell lines are homogenous clonal cells, whereas tumors are comprised of heterogeneous cell populations. While the functional significance of the loss of p53 functions in tumor cells has been extensively studied, recent studies have also shown the tumor suppressive role of p53 in stroma cells and tumor-infiltrating immune cells ${ }^{37-42}$. It remains to be determined to what degree the actual tumor cells, tumor-associating stroma cells, and tumor-infiltrating immune cells contribute to the TP53 signature.

In conclusion, our study shows that PDSs are a reliable prognostic predictor of clinical outcomes in the majority of cancer types and treatments. Moreover, PDSs predict clinical outcomes better than the analysis of 
A

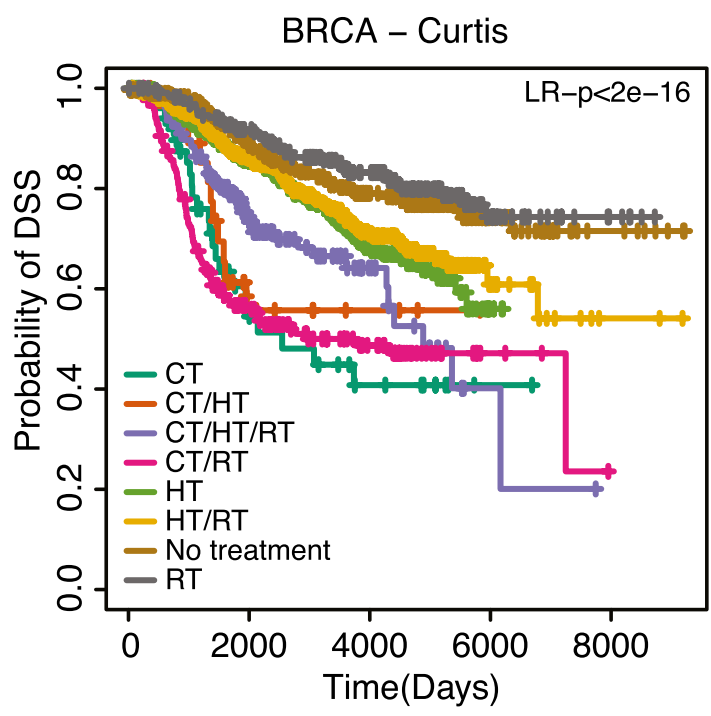

B

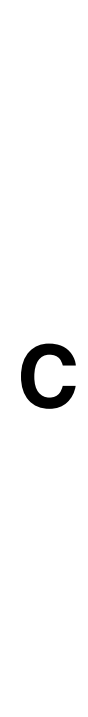

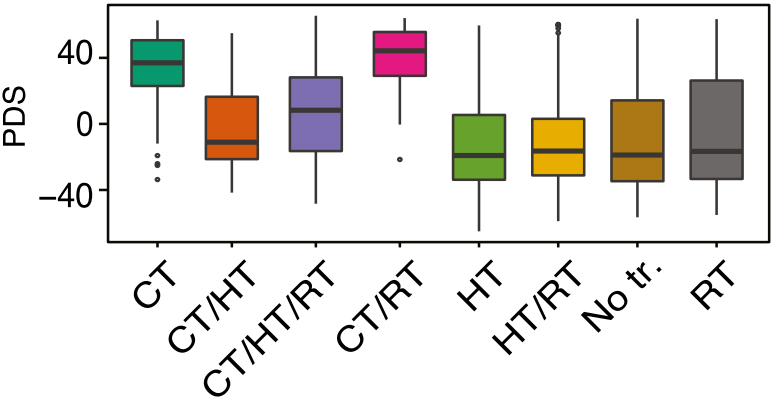

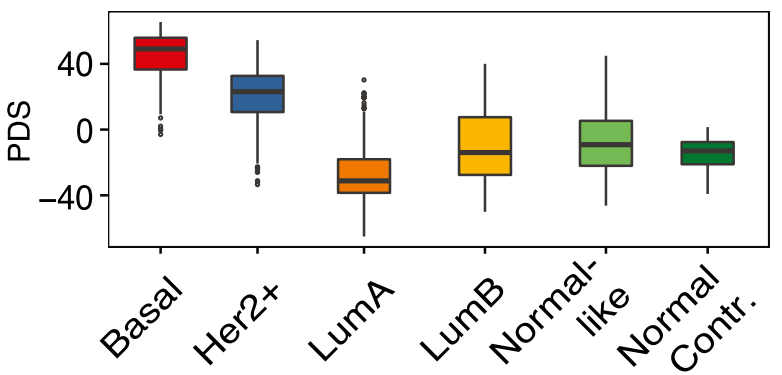

D
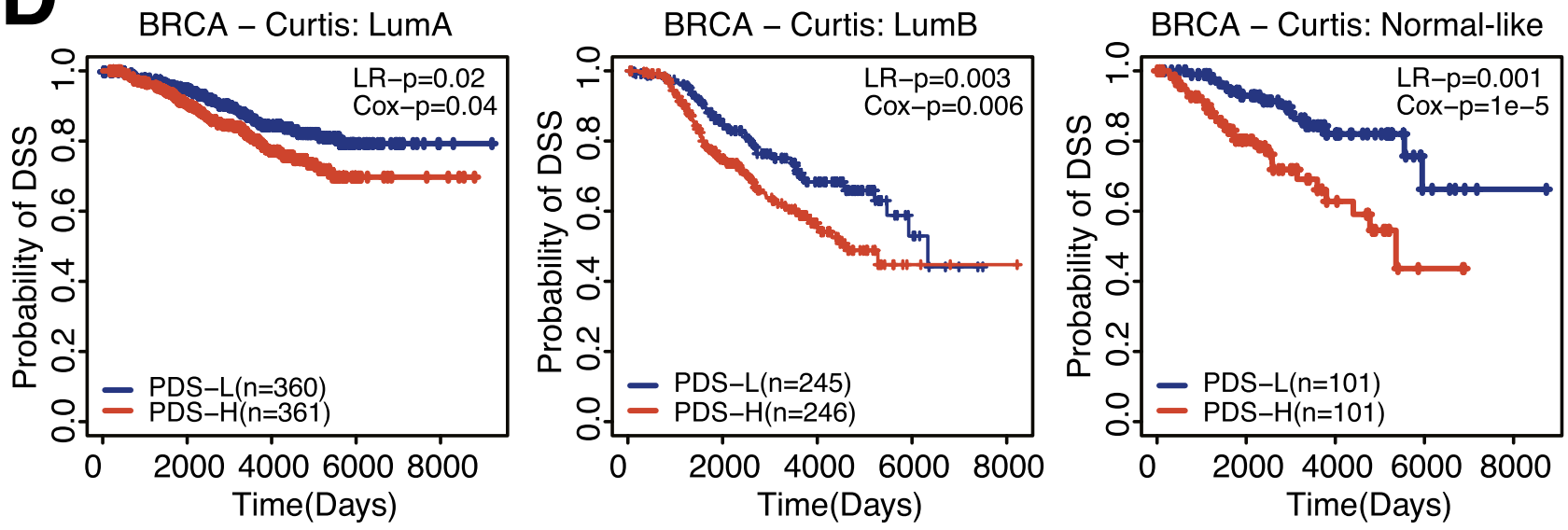

Figure 5. PDSs in breast cancer. (A) Differences in survival comparing different treatment strategies in the METABRIC/Curtis dataset. LR-p p-value calculated between TP53 groups using Log-rank test, DSS diseasespecific survival. (B) PDSs compared between distinct treatment groups. (C) PDSs for breast cancer subtypes in METABRIC/Curtis dataset. (D) KM plots evaluating the association between PDS and survival in the METABRIC/Curtis dataset within PAM50 breast cancer subtypes. $L R-p$ p-value calculated between TP53 groups using Log-rank test, Cox- $p$ p-value calculated using Coxph regression using PDSs as continuous variable, DSS disease-specific survival.

TP53 mutation status. In combination with another gene signature or mutation status, the TP53 signature may be used to predict responses to certain therapies more accurately and may help to select a better therapeutic option in certain cancers.

\section{Methods}

All data presented in this paper are publicly available at http://firebrowse.org (TCGA), https://precog.stanford. edu (PRECOG), https://portals.broadinstitute.org/ (CCLE), or Curtis et al. ${ }^{17}$.

TCGA. Level 3 RNA-seq data from The Cancer Genome Atlas (TCGA) project were downloaded from FireBrowse (http://firebrowse.org). RSEM-normalized gene expression data for 20,501 genes was provided ${ }^{43}$. Only cancer types containing at least 20 patients with protein-altering TP53 mutations and at least a $10 \%$ proteinaltering TP53 mutation rate were included, resulting in the inclusion of 20 cancer types. These included KICH, kidney chromophobe; LGG, brain lower grade glioma; GBM, glioblastoma multiforme; BRCA, breast invasive carcinoma; LUSC, lung squamous cell carcinoma; LUAD, lung adenocarcinoma; READ, rectum adenocarcinoma; COAD, colon adenocarcinoma; UCS, uterine carcinosarcoma; UCEC, uterine corpus endometrial carcinoma; OV, ovarian serous cystadenocarcinoma; HNSC, head and neck squamous carcinoma; PRAD, prostate adenocarcinoma; STAD, stomach adenocarcinoma; SKCM, skin cutaneous melanoma; BLCA, bladder urothe- 
lial carcinoma; LIHC, liver hepatocellular carcinoma; SARC, sarcoma; PAAD, pancreatic adenocarcinoma; and ESCA, esophageal carcinoma (Table 1). TCGA somatic mutation information and clinical data were also downloaded from FireBrowse (http://firebrowse.org).

PRECOG. Gene expression datasets were obtained from PREdiction of Clinical Outcomes from Genomic profiles (PRECOG, https://precog.stanford.edu) ${ }^{15}$ as normalized datafiles. Clinical data including overall survival times and outcomes for studies were obtained from the same resource. Datasets were filtered by tissue type to match TCGA tumor types, and only datasets with more than 40 samples and over $20 \%$ mortality rate were included in our analysis. This resulted in the inclusion of 126 datasets.

METABRIC/Curtis data. Breast cancer data for the METABRIC/Curtis dataset was downloaded from a previous publication ${ }^{17}$. Disease-specific survival and treatment information was obtained from the same publication.

CCLE. The Cancer Cell Line Encyclopedia (CCLE) data was downloaded from the CCLE database (https:// portals.broadinstitute.org/ $/)^{16}$. This dataset provides gene expression profiles measured by microarray experiments for more than 1100 cancer cell lines and their sensitivity to 24 anticancer drugs. Drug Activity Area was utilized as assessment of drug efficacy, where higher Drug Activity Areas indicate higher sensitivity.

Generation of PDS signatures. For a detailed explanation of PDS signature generation, see Zhao et al. ${ }^{14}$. Briefly, for each cancer type, patients were separated into two groups based on the presence or absence of protein-altering TP53 mutations. A logistic regression model was fitted for each gene using expression as the independent variable and TP53 mutation status $(0=$ TP53 wild type, $1=$ TP53 mutation $)$ as the dependent variable. Based on the resultant $p$-values and $\beta$-coefficients, genes were separated into an up profile $(\beta>0)$ and a down $(\beta<0)$ profile. For both up and down profiles, p-values were - $\log 10$ transformed, trimmed at 10 to reduce outliers, and scaled into $[0,1]$ to obtain the weights for genes. The resulting up and down profiles constituted the p53 deficiency signature for a cancer type.

Calculation of PDSs. For a detailed explanation of p53 deficiency inference, see Zhao et al. ${ }^{14}$. Briefly, the enrichment of cancer type-specific p53 deficiency signatures in patient samples was determined by BASE ${ }^{44}$, a rank-based gene set enrichment method, for each utilized gene expression dataset. BASE calculated sample-specific scores, named PDSs based on their gene expression profiles measured by microarray or RNA-seq analysis. The deficiency scores indicated the degree of p53 pathway deficiency, with a higher score indicating lower p53 activity.

Survival analysis. Survival analyses were performed using the R survival package (version 3.2-7). Log-rank tests were performed to evaluate overall or disease-specific survival probabilities between two groups using the 'survdiff' function. Univariate Coxph regression was performed on continuous PDSs using the 'coxph' function. Kaplan-Meier plots were generated using the 'survfit' function. For KM plots using the TCGA data, the percentage of TP53 mutated samples was used as a cutoff to determine low and high PDSs. This was done to allow for a direct comparison between the prognostic significance of TP53 mutations and PDSs without the confounding of different group sizes. For all other KM plots, the median PDS was used to separate low and high PDS groups.

For the survival analysis in the PRECOG dataset, PDSs were inferred in a cancer type-specific manner. After computing the PDSs, we fitted a univariate Cox regression model to measure the association between PDSs and all-cause or disease-specific mortality (if available). Z-scores were extracted from the fitted models and a meta $\mathrm{z}$-score was calculated for PDSs across microarray datasets of the same cancer type. A meta $\mathrm{z}$-score was calculated using the weighted Stouffer's z-score method which uses the dataset sample size as weights. Meta p-values were calculated from the meta $\mathrm{z}$-scores by referring to the standard normal distribution.

Cell culture. The HCC-1954, HCC-202, AU-565, SKBR-3, BT-474, and T47D cell lines (ATCC) were maintained in RPMI media (Corning) supplemented with 10\% fetal bovine serum (FBS) and 1\% penicillin-streptomycin (Corning), whereas MDA-MB-468 and HCC-1419 (ATCC) were cultured in RPMI with 10\% FBS, 1\% penicillin-streptomycin (HyClone), $1 \%$ MEM non-essential amino acids (Gibco), and $100 \mu \mathrm{M}$ sodium pyruvate (Gibco). The MDA-MB-231 cell line (ATCC) was cultured in DMEM (Corning) with 10\% FBS and 1\% penicillin-streptomycin (Corning). MTS assays were performed using the CellTiter 96 Aqueous One Solution Cell Proliferation Assay kit (Promega) as previously described ${ }^{45}$. In brief, 10,000 cells/well were plated into a 96 well plate. Twenty-four hours later, the cells were treated with paclitaxel (LC Laboratories) for an additional $48 \mathrm{~h}$. DMSO and the apoptosis-inducing pan-kinase inhibitor staurosporine $(1 \mu \mathrm{M})$ were used as negative and positive controls, respectively. The absorbance at $490 \mathrm{~nm}$ (OD $490 \mathrm{~nm}$ ) was normalized against DMSO.

Statistical analysis. The Spearman correlation coefficient was reported for correlation analyses and was calculated using the R 'cor' function. Area Under the Curve (AUC) values evaluating the ability of PDSs in predicting TP53 mutation status in the CCLE dataset were calculated and plotted using the 'pROC' R package. The statistical difference between Drug Activity Areas between TP53 deficiency groups in the CCLE dataset was calculated by two-sided Student's t-tests. 
Received: 19 October 2021; Accepted: 3 January 2022

Published online: 25 January 2022

\section{References}

1. Vousden, K. \& Prives, C. p53 and prognosis new insights and further complexity. Cell 120, 7-10 (2005).

2. Bailey, M. H. et al. Comprehensive characterization of cancer driver genes and mutations. Cell 173, 371-385 (2018).

3. Malkin, D. p53 and the Li-Fraumeni syndrome. Cancer Genet. Cytogen. 66, 83-92 (1993).

4. Donehower, L. A. et al. Mice deficient for p53 are developmentally normal but susceptible to spontaneous tumours. Nature 356, 215-221 (1992).

5. Robles, A. I. \& Harris, C. C. Clinical outcomes and correlates of TP53 mutations and cancer. Cold Spring Harb. Perspect. Biol. 2, a001016 (2010).

6. Russo, A. et al. The TP53 Colorectal cancer international collaborative study on the prognostic and predictive significance of p53 mutation: Influence of tumor site, type of mutation, and adjuvant treatment. J. Clin. Oncol. 23, 7518-7528 (2005).

7. Børresen-Dale, A. TP53 and breast cancer. Hum. Mutat. 21, 292-300 (2003).

8. Zhang, W., Edwards, A., Flemington, E. K. \& Zhang, K. Significant prognostic features and patterns of somatic TP53 mutations in human cancers. Cancer Inform. 16, 117693511769126 (2017).

9. Ueno, H. et al. Landscape of driver mutations and their clinical impacts in pediatric B-cell precursor acute lymphoblastic leukemia. Blood Adv. 4, 5165-5173 (2020).

10. Martinez-Rivera, M. \& Siddik, Z. H. Resistance and gain-of-resistance phenotypes in cancers harboring wild-type p53. Biochem. Pharmacol. 83, 1049-1062 (2012).

11. Wasylishen, A. R. \& Lozano, G. Attenuating the p53 pathway in human cancers: Many means to the same end. Cold Spring Harb. Perspect. Med. 6, a026211 (2016).

12. París, R., Henry, R. E., Stephens, S. J., McBryde, M. \& Espinosa, J. M. Multiple p53-independent gene silencing mechanisms define the cellular response to p53 activation. Cell Cycle 7, 2427-2433 (2008).

13. Roman-Gomez, J. et al. $5^{\prime} \mathrm{CpG}$ island hypermethylation is associated with transcriptional silencing of the p21 ${ }^{\mathrm{CIP} 1 / \mathrm{WAF} 1 / \mathrm{SDI} 1}$ gene and confers poor prognosis in acute lymphoblastic leukemia. Blood 99, 2291-2296 (2002).

14. Zhao, Y. et al. A p53-deficiency gene signature predicts recurrence risk of patients with early stage lung adenocarcinoma. Cancer Epidemiol. Prev. Biomark. 27, 86 (2017).

15. Gentles, A. J. et al. The prognostic landscape of genes and infiltrating immune cells across human cancers. Nat. Med. 21, 938-945 (2015).

16. Barretina, J. et al. The Cancer Cell Line Encyclopedia enables predictive modelling of anticancer drug sensitivity. Nature 483, 603-607 (2012).

17. Curtis, C. et al. The genomic and transcriptomic architecture of 2,000 breast tumours reveals novel subgroups. Nature 486, 346-352 (2012).

18. Koboldt, D. C. et al. Comprehensive molecular portraits of human breast tumours. Nature 490, 61-70 (2012).

19. Jost, C. A. \& Marin, M. C. p73 is a human p53-related protein that can induce apoptosis. Nature 389, 191-194 (1997).

20. Yang, A. et al. p63, a p53 homolog at 3q27-29, encodes multiple products with transactivating, death-inducing, and dominantnegative activities. Mol. Cell 2, 305-316 (1998).

21. Laurenzi, V. \& Melino, G. Evolution of functions within the p53/p63/p73 family. Ann. N.Y. Acad. Sci. 926, 90-100 (2000).

22. Miller, L. D. et al. An expression signature for p53 status in human breast cancer predicts mutation status, transcriptional effects, and patient survival. Proc. Natl. Acad. Sci. U.S.A. 102, 13550-13555 (2005).

23. van 't Veer, L. J. et al. Gene expression profiling predicts clinical outcome of breast cancer. Nature 415, 530-536 (2002).

24. Sotiriou, C. et al. Breast cancer classification and prognosis based on gene expression profiles from a population-based study. Proc. Natl. Acad. Sci. 100, 10393-10398 (2003).

25. Chipidza, F. E. et al. Development and validation of a novel TP53 mutation signature that predicts risk of metastasis in primary prostate cancer. Clin. Genitourin Cancer. 9, 246-254.e5 (2020).

26. Caponio, V. C. A. et al. Computational analysis of TP53 mutational landscape unveils key prognostic signatures and distinct pathobiological pathways in head and neck squamous cell cancer. Br. J. Cancer 123, 1302-1314 (2020).

27. Wang, K. et al. Prognostic value of MGMT promoter methylation and TP53 mutation in glioblastomas depends on IDH1 mutation. Asian Pac. J. Cancer Prev. 15, 10893-10898 (2015).

28. Shahbandi, A., Nguyen, H. D. \& Jackson, J. G. TP53 mutations and outcomes in breast cancer: Reading beyond the headlines. Trends Cancer 6, 98-110 (2020).

29. Coutant, C. et al. Distinct p53 gene signatures are needed to predict prognosis and response to chemotherapy in ER-positive and ER-negative breast cancers. Clin. Cancer Res Off. J. Am. Assoc. Cancer Res. 17, 2591-2601 (2011).

30. Wang, Y. et al. TP53 mutations are associated with higher rates of pathologic complete response to anthracycline/cyclophosphamide-based neoadjuvant chemotherapy in operable primary breast cancer. Int. J. Cancer 138, 489-496 (2016).

31. Glück, S. et al. TP53 genomics predict higher clinical and pathologic tumor response in operable early-stage breast cancer treated with docetaxel-capecitabine \pm trastuzumab. Breast Cancer Res. Treat. 132, 781-791 (2012).

32. Kim, T. et al. Predictive significance of p53, Ki-67, and Bcl-2 expression for pathologic complete response after neoadjuvant chemotherapy for triple-negative breast cancer. J. Breast Cancer 18, 16-21 (2015).

33. Chen, M.-B. et al. Value of TP53 status for predicting response to neoadjuvant chemotherapy in breast cancer: A meta-analysis. PLoS ONE 7, e39655 (2012).

34. Bertheau, P. et al. Exquisite sensitivity of TP53 mutant and basal breast cancers to a dose-dense epirubicin-cyclophosphamide regimen. PLoS Med. 4, e90 (2007).

35. Jackson, J. G. et al. p53-mediated senescence impairs the apoptotic response to chemotherapy and clinical outcome in breast cancer. Cancer Cell 21, 793-806 (2012).

36. Ungerleider, N. A. et al. Breast cancer survival predicted by TP53 mutation status differs markedly depending on treatment. Breast Cancer Res. 20, 115 (2018).

37. Thorsson, V. et al. The immune landscape of cancer. Immunity 48, 812-830 (2018).

38. Bar, J., Moskovits, N. \& Oren, M. Involvement of stromal p53 in tumor-stroma interactions. Semin. Cell Dev. Biol. 21, 47-54 (2010).

39. Arandkar, S. et al. Altered p53 functionality in cancer-associated fibroblasts contributes to their cancer-supporting features. Proc. Natl. Acad. Sci. 115, 201719076 (2018).

40. Pilley, S., Rodriguez, T. A. \& Vousden, K. H. Mutant p53 in cell-cell interactions. Gene Dev. 35, 433-448 (2021).

41. Cui, Y. \& Guo, G. Immunomodulatory function of the tumor suppressor p53 in host immune response and the tumor microenvironment. Int. J. Mol. Sci. 17, 1942 (2016).

42. Guo, G., Yu, M., Xiao, W., Celis, E. \& Cui, Y. Local activation of p53 in the tumor microenvironment overcomes immune suppression and enhances antitumor immunity. Cancer Res. 77, 2292-2305 (2017).

43. Li, B. \& Dewey, C. N. RSEM: Accurate transcript quantification from RNA-Seq data with or without a reference genome. BMC Bioinform. 12, 323 (2011). 
44. Cheng, C., Yan, X., Sun, F. \& Li, L. M. Inferring activity changes of transcription factors by binding association with sorted expression profiles. BMC Bioinform. 8, 452 (2007).

45. Canfield, K. et al. Receptor tyrosine kinase ERBB4 mediates acquired resistance to ERBB2 inhibitors in breast cancer cells. Cell Cycle (Georgetown) 14, 648-655 (2015).

\section{Acknowledgements}

We would like to thank Mr. Scott Bang for critical reading of the manuscript. We also thank Dr. Loral Showalter for valuable advice on breast cancer cell culture. This study is supported by the Farris Family Foundation Grant (to MK), the Cancer Prevention Research Institute of Texas (CPRIT) (RR180061 to CC), the National Cancer Institute of the National Institutes of Health (R21CA227996 to CC, R00CA140948, R03CA208384, and R03CA230828 to MK), and the T32 training Grant of the National Institutes of Health (T32AI007363 to ES). CC is a CPRIT Scholar in Cancer Research. MK is a recipient of the Mary Kay Foundation research grant. ET is a recipient of the 2020 Summer Undergraduate Research Experience (SURE) Fellowship at Kent State University. We would also like to thank the Department of Biological Sciences at Kent State University for their support. MK dedicates this paper to the memory of Neil Spector at Duke University.

\section{Author contributions}

E.S., C.C. and M.K. conceived the study and designed the experiments. C.C. and M.K. supervised the entire study. E.T. and S.K. performed the cell culture experiments. E.S. and C.C. carried out dataset analysis. E.S., E.T., S.K., C.C. and M.K. analyzed the data and wrote the manuscript. All authors reviewed the manuscript.

\section{Competing interests}

The authors declare no competing interests.

\section{Additional information}

Correspondence and requests for materials should be addressed to C.C. or M.K.

Reprints and permissions information is available at www.nature.com/reprints.

Publisher's note Springer Nature remains neutral with regard to jurisdictional claims in published maps and institutional affiliations.

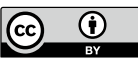

Open Access This article is licensed under a Creative Commons Attribution 4.0 International License, which permits use, sharing, adaptation, distribution and reproduction in any medium or format, as long as you give appropriate credit to the original author(s) and the source, provide a link to the Creative Commons licence, and indicate if changes were made. The images or other third party material in this article are included in the article's Creative Commons licence, unless indicated otherwise in a credit line to the material. If material is not included in the article's Creative Commons licence and your intended use is not permitted by statutory regulation or exceeds the permitted use, you will need to obtain permission directly from the copyright holder. To view a copy of this licence, visit http://creativecommons.org/licenses/by/4.0/.

(C) The Author(s) 2022 